\title{
METASTRONGYLUS MADAGASCARIENSIS n. sp., QUATRIÈME ESPÈCE DE STRONGLE PULMONAIRE CHEZ LE PORC DOMESTIQUE
}

\author{
Par Alain G. CHABAUD et Simon GRÉtillat
}

La bronchopneumonie vermineuse du porc, maladie grave, tuant souvent les animaux jeunes, est due à la présence dans les bronches de certains Strongles qui appartiennent au genre Metastrongylus Molin 1861. On connait actuellement trois espèces différentes, probablement cosmopolites. Il a été trouvé dans les bronches d'un porcelet, à Tananarive, en juin 1956, une autre espèce nettement différente des précédentes. On peut donc supposer que cette quatrième espèce est spéciale à Madagascar, car la faune helminthologique du porc a été déjà très étudiée, et il est probable qu'une espèce aussi facilement visible serait déjà connue, si elle était cosmopolite.

\section{Description}

La description suivante porte sur 6 femelles et 5 mâles en bon état et sur plusieurs fragments des deux sexes.

Corps filiforme, très atténué en avant et n'atteignant sa largeur maxima que dans la partie toute postérieure. Cuticule très fine, parfois légèrement plissée, dépourvue de stries transversales. Pas d'ailes latérales. Bouche presque circulaire ; les deux lèvres latérales trilobées, qui sont habituelles aux espèces du genre, sont ici à peine indiquées (1) et sont très plates (fig. $1 \mathrm{~A}$ ).

Les papilles céphaliques comprennent :

— un cycle interne formé de 6 très petites papilles, au sommet de chacun des lobes labiaux ;

(1) Un spécimen étudié en vue apicale présente, au contraire, des lèvres très bien marquées (fig. $1 \mathrm{~B}$ ), alors que tous les autres exemplaires ont des lèvres plates. Comme il s'agit d'un court fragment antérieur, la détermination est impossible et il s'agit peut-être d'une autre espèce.

ANn. de Parasitologie, T. XXXI, $x^{\circ} 5-6 .-1956$. 
- un cycle externe formé de 4 papilles médio-médianes, de 4 papilles latéro-médianes (groupées 2 à 2 ) et de 2 papilles ventrolatérales (situées un peu en arrière des amphides).

Les 10 papilles du cycle externe sont très petites, mais ont néanmoins un aspect nettement sétiforme (fig. $1 \mathrm{C}$ ). Cavité buccale très courte. Esophage faiblement claviforme. Anneau nerveux situé un peu en arrière du tiers antérieur de l'œsophage; pore excréteur et diérides un peu en arrière du tiers postérieur. Les diérides sont très petites, mais elles sont incluses dans une légère vésicule cuticulaire. Intestin à peu près aussi large que l'œsophage. Rectum remarquablement grand, formant une cavité cylindrique à parois épaisses, haute de 200 à $400 \mu$ et large de $100 \mu$.
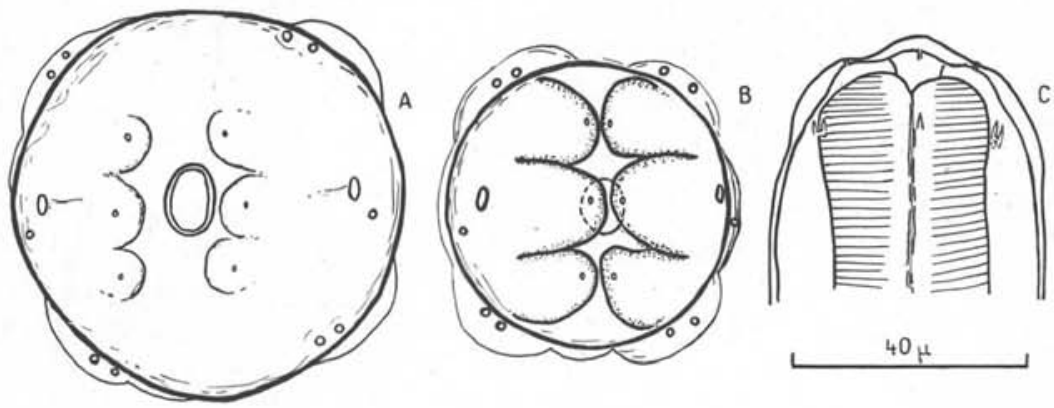

Fig. 1. - A : Metastrongylus madagascariensis, femelle. Extrémité céphalique ; vue apicale.

B : Metastrongylus sp. Vue apicale d'un fragment non déterminable, appartenant probablement à une autre espèce à lèvres bien marquées.

C : Metastrongylus madagascariensis, mâle. Extrémité céphalique ; vue latérale.

Femelle. - Corps long de 22 à $26 \mathrm{~mm}$., large au maximum de $250 \mu$. Chez une femelle de $26 \mathrm{~mm}$., l'œsophage est long de $430 \mu$. L'anneau nerveux, les diérides et le pore excréteur sont à $215 \mu$, $270 \mu$ et $280 \mu$ de l'apex. (L'œsophage atteint une longueur exceptionnelle de $520 \mu$ chez une femelle longue de $24 \mathrm{~mm}$.). Extrémité postérieure enflée, puis brusquement rétrécie au niveau de la vulve (fig. $2 \mathrm{C}$ ). En avant de celle-ci existe un provagin, qui est très différent de celui des autres espèces et se rapproche plutôt de celui de certains Protostrongylus. Il est formé par une lame cuticulaire mince, étendue devant la vulve et se terminant distalement par un gland ovoïde appliqué contre l'anus, ou placé dans la cavité formée par la pointe caudale et le début de la région vulvaire (fig. 3, A, B). 
La cuticule qui forme le provagin n'est pas enflée en vésicule et la largeur totale de l'organe ne dépasse pas $150 \mu$ (1).

L'ovéjecteur figuré en $2 \mathrm{~B}$ a les caractères habituels au genre (cf. Dougherty, 1949, fig. 1) ; il est long de $550 \mu$. Les œufs sont longs de $60 \mu$ et larges de $42 \mu$. La larve est repliée dans une fine

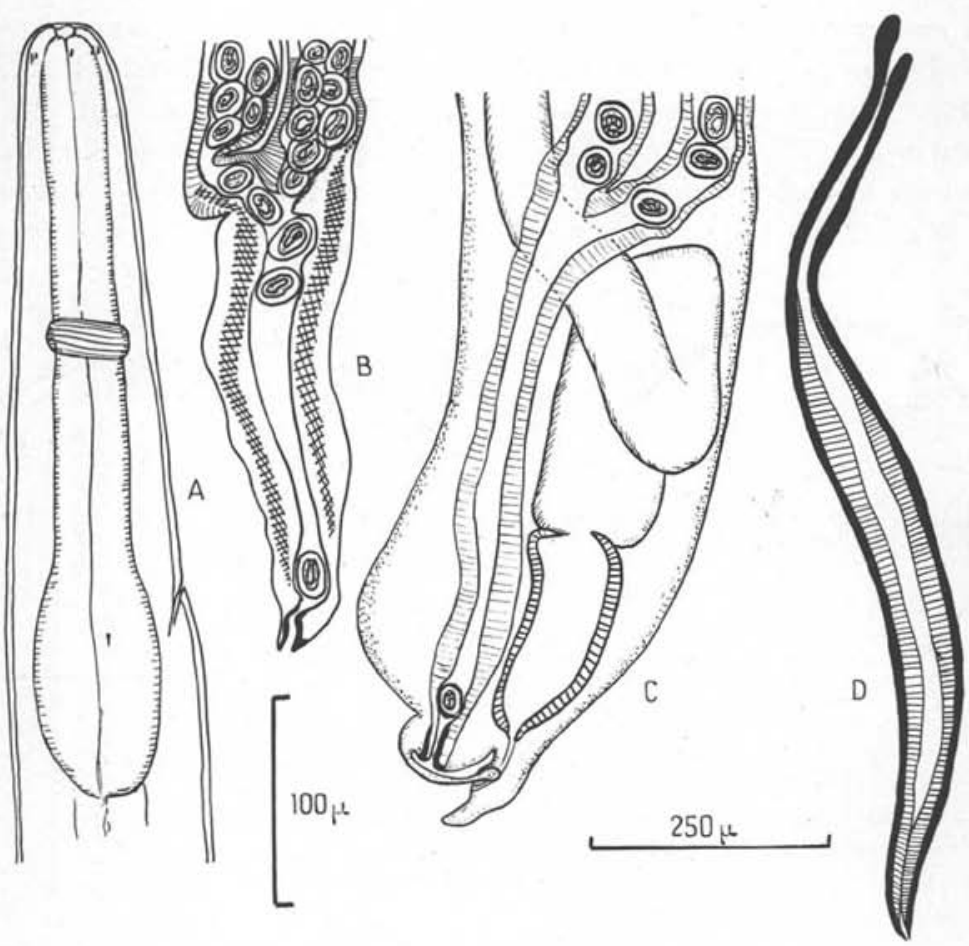

FiG. 2. - Metastrongylus madagascariensis.

A : Extrémité antérieure du mâle. Vue latérale. Echelle 0-100 $\mu$.

B : Dissection de l'ovéjecteur. Echelle 0-250 $\mu$.

C : Extrémité postérieure d'une femelle. Vue latérale. Echelle 0-250 $\mu$.

D : Spicules en vue ventrale. Echelle 0-250 u.

membrane interne qui l'isole largement de la coque externe. Dorsalement à la région vulvaire, le corps se termine par un mucron long de $85 \mu$. L'anus s'ouvre sur la face ventrale de ce mucron, et la queue proprement dite ne mesure que $50 \mu$.

(1) Ainsi que le remarque Gedoelst, ces curieuses formations représentent probablement des ceintures de chasteté, qui empêchent les nouvelles copulations chez les femelles mûres. 
Mâle. - Corps long de 9,5 à $12 \mathrm{~mm}$., large de $160 \mu$. Les principales dimensions d'un mâle long de $11 \mathrm{~mm}$. sont les suivantes : œsophage : $370 \mu$, anneau nerveux, diérides et pore excréteur respectivement à $200 \mu, 255 \mu$ et $270 \mu$ de l'extrémité antérieure (fig. $2 \mathrm{~A}$ ). Spicules à pointe simple, sans crochet, longs de $1.050 \mu$ (fig. 2 D). Gubernaculum très fortement concave ventralement, long de $60 \mu$. La bourse caudale s'étend perpendiculairement à l'axe du corps; ses caractères sont indiqués sur les figures $3 \mathrm{C}, 4 \mathrm{~A}$ et $4 \mathrm{~B}$.

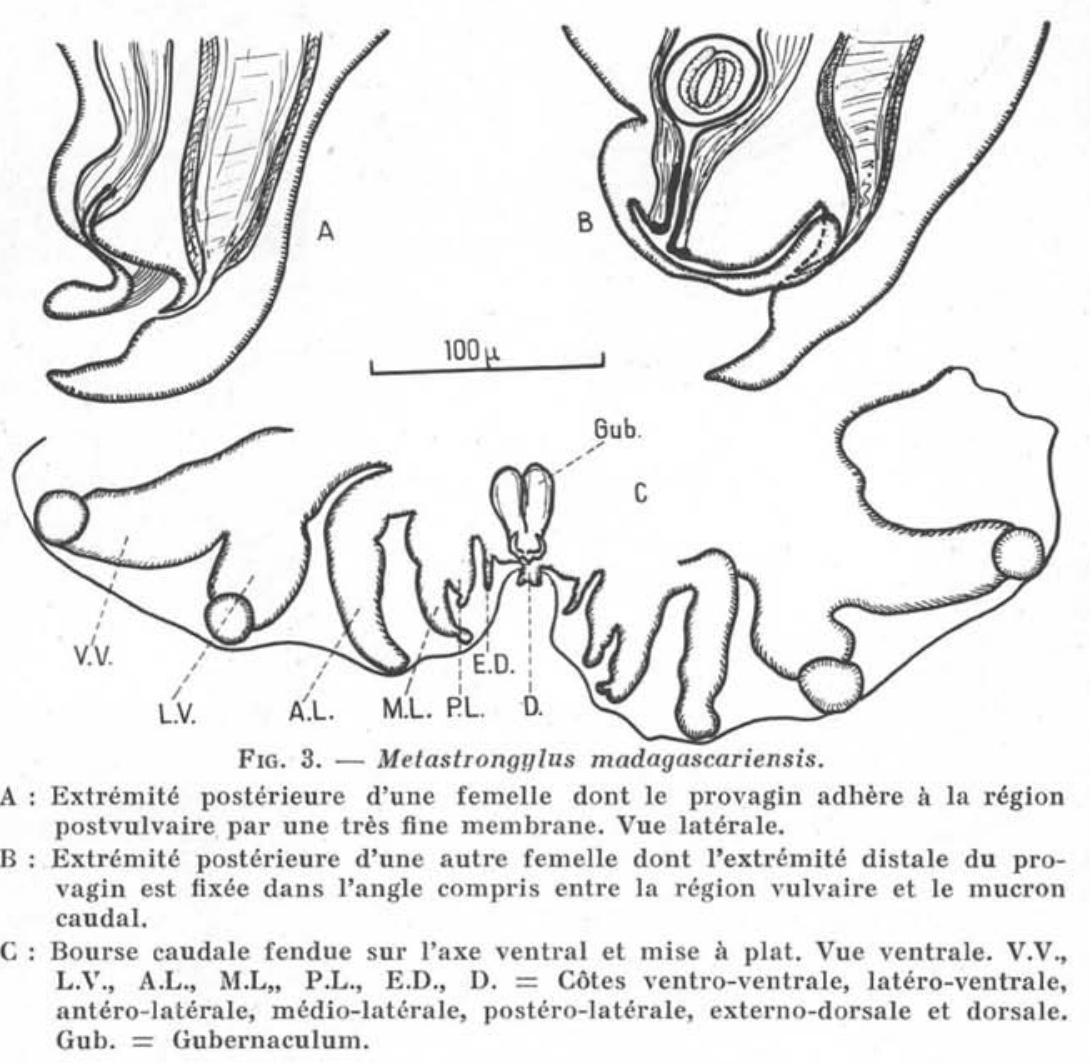

\section{Discussion}

La détermination des trois espèces déjà connues chez les Metastrongylus est rendue facile grâce aux publications de Gedoelst (1923) et de Dougherty (1944). 


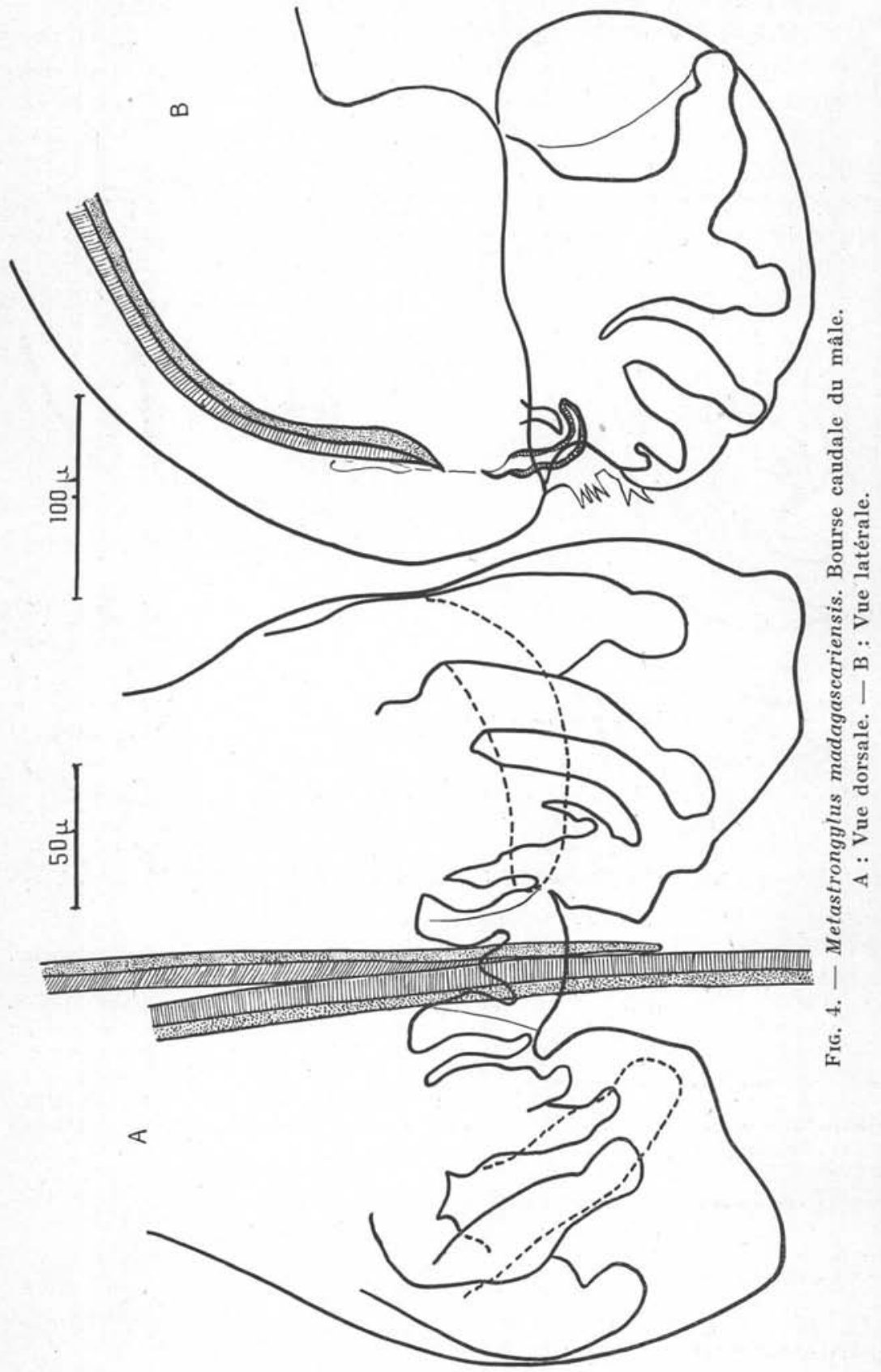


Notre espèce n'a aucune affinité avec $M$. apri (Gmelin 1790) ou M. salmi Gedoelst 1923, mais se rapproche au contraire de $M$. $p u$ dentotectus Vostokov 1905 (= M. brevivaginatus). Elle s'en distingue :

1) par les dimensions plus faibles. Mâle de $11 \mathrm{~mm}$. au lieu de $16 \mathrm{~mm}$. Spicules de $1 \mathrm{~mm}$., au lieu de $1,4 \mathrm{~mm}$;

2) par la pointe des spicules, qui est simple et aiguë, et n'a pas de crochet ;

3) par les caractères de la bourse caudale, et en particulier par la côte ventro-ventrale, qui est presque perpendiculaire à la côte latéro-ventrale ;

4) par le pro-vagin, qui ne forme pas une bulle vésiculeuse en avant de la vulve, mais seulement une lame mince terminée par un renflement ovoïde (les femelles ont des œufs bien formés, et ne peuvent être considérées comme juvéniles) ;

5) par les lèvres céphaliques, à peine indiquées, et très plates.

Nous pensons donc que l'espèce est nouvelle et proposons de la désigner sous le nom de Metastrongylus madagascariensis n. sp.

Hôte type: Sus scrofa scrofa Linné.

Localisation : Bronches.

Lieu d'origine: Tananarive (Madagascar).

\section{RÉSU MÉ}

Le genre Metastrongylus comprend trois espèces cosmopolites, parasites dans les bronches des Suidés. Nous décrivons une quatrième espèce trouvée chez un porc domestique à Madagascar. L'espèce la plus proche est $M$. pudentotectus, mais elle s'en sépare aisément par différents caractères : bourse caudale et spicule chez le mâle, forme du provagin chez la femelle.

\section{RÉFÉRENCES}

Dougherty (E. C.), 1944. - The genus Metastrongylus Molin, 1861 (Nematoda : Metastrongylide). Proc. Helm. Soc. Wash., XI, 66-72, fig. A-F.

- 1949. - The phylogeny of the Nematode family Metastrongylida Leiper (1909) : a correlation of host and symbiote evolution. Parasit., XXXIX, 222-234, fig. 1-27.

Gedoelst (L.), 1923. - Le genre Metastrongylus Molin, 1861. Bull. Soc. Path. Exot., XVI, 622-630, fig. 1-4.

Institut de Parasitologie de la Faculté de Médecine de Paris et Laborat. central de l'Elevage et des Industries animales, Tananarive. 